\title{
Implementación y evaluación de un protocolo de analgesia multimodal en el reemplazo total primario de rodilla que incluye bloqueo de canal de aductores guiado por ultrasonografía más infiltración de anestésico local periarticular
} Implementation and evaluation of a multimodal analgesia protocol for total knee arthroplasty including ultrasoundguided adductor canal block and periarticular local anesthetic infiltration

L. M. Zamudio-Castilla1,2, J. F. González Vera², J. R. Rodríguez-Conde² y F. C. Dorado-Velasco

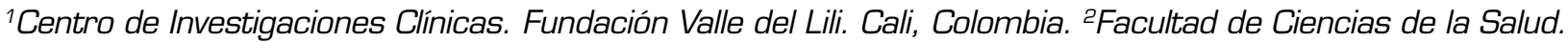
Universidad Icesi. Cali, Colombia. ${ }^{3}$ Departamento de Anestesiología. Fundación Valle del Lili. Cali, Colombia

\section{RESUMEN}

Introducción: La anestesia regional guiada por ultrasonografía es una técnica segura y efectiva para el manejo del dolor postoperatorio. Este estudio evalúa la implementación de un protocolo analgésico para el reemplazo total primario de rodilla (RTPR).

Métodos: Estudio observacional ambispectivo de cohortes no aleatorizado, realizado en un grupo de pacientes llevados a RTPR que recibieron infiltración de anestésico local periarticular más bloqueo del canal de aductores (IAL+BCA) como parte de un nuevo protocolo analgésico, frente a un grupo previo que recibió catéter perineural femoral más bloqueo del nervio ciático [CPF+BNC). La valoración de la intensidad del dolor se realizó utilizando la escala verbal numérica (EVN). El desenlace principal fue el dolor postoperatorio inmediato a las 24 y 48 horas. Se evaluó el cumplimiento de metas de rehabilitación física a las 48 horas como desenlace secundario.

Resultados: Se analizaron 112 pacientes (67 en $I A L+B C A$ y 45 en CPF + BNC). Ambas poblaciones

Zamudio-Castilla LM, González Vera JF, Rodríguez-Conde JR, DoradoVelasco FC. Implementación y evaluación de un protocolo de analgesia multimodal en el reemplazo total primario de rodilla que incluye bloqueo de canal de aductores guiado por ultrasonografía más infiltración de anestésico local periarticular. Rev Soc Esp Dolor. 2021;28(1):9-18

\section{ABSTRACT}

Background: Ultrasound-guided regional anesthesia is a safe and effective technique in postoperative pain management. This study evaluates the implementation an analgesic protocol for total knee arthroplasty (TKA) including different nerve blocks.

Methods: An observational ambispective non randomized cohort study was performed between a group of patients undergoing TKA who received local infiltration analgesia plus adductor canal block (LIA+ACB) as part of a new analgesic protocol against a previous group who received perineural femoral catheter plus sciatic nerve block (PFC+SNB). The measurement of pain intensity was made using the numerical rating scale (NRS). The main outcome was the immediate postoperative pain, at 24 and 48 hours. The fulfillment of physical rehabilitation goals was evaluated as a secondary outcome.

Results: 112 patients were analyzed ( 67 in $\mathrm{LIA+ACB}$ and 45 in PFC+SNB). Both groups were comparable in demographic characteristics. The median pain at rest on the first and second postoperative days was NRS

Recibido: 23-09-2020 Aceptado: 14-02-2021

Correspondencia: Fabián Camilo Dorado-Velasco fabian.dorado@fvl.org.co 
fueron comparables en variables demográficas. La mediana del dolor en reposo en el primer y segundo día postoperatorios fue EVN 2/10 para ambos grupos. El promedio del dolor en movimiento en el segundo día postoperatorio fue EVN 5/10 para los pacientes con $\mathrm{IAL}+\mathrm{BCA}$ y $4 / 10$ para los pacientes con CPF + BNC, $p=0,073$. El porcentaje de pacientes que cumplieron el $80 \%$ o más de las metas de rehabilitación fue similar en ambos grupos $(p=0,201)$.

Conclusiones: Ambas técnicas son equivalentes en el manejo analgésico postoperatorio del RTPR durante las primeras 48 horas. A pesar de que es conocido que la técnica de IAL + BCA genera menos compromiso motor del cuádriceps, esto no se reflejó en un mejor desempeño durante la rehabilitación física, posiblemente por un insuficiente control analgésico a las 48 horas.

Palabras clave: Artroplastia, rodilla, anestesia y analgesia, dolor posoperatorio, medicina física y rehabilitación.

\section{INTRODUCCIÓN}

El reemplazo total primario de rodilla (RTPR) es un procedimiento cada vez más frecuente para el tratamiento de enfermedad articular degenerativa, en su mayoría de tipo osteoartritis, caracterizada por dolor, limitación funcional y afectación en la calidad de vida principalmente en población mayor de 65 años $(1,2)$. Según Inacio y cols., quienes realizaron una proyección sobre la incidencia de RTPR basada en datos epidemiológicos poblacionales en los Estados Unidos, se prevé un aumento en el número de esta de aproximadamente un $143 \%$ para 2050, en comparación con el año 2012 [ㄱ] , acorde con el proceso de envejecimiento de la sociedad.

Ante este aumento en la realización de RTPR, y siendo el dolor y la incapacidad los principales síntomas para su indicación, se han canalizado esfuerzos hacia lograr un adecuado control analgésico con una rápida recuperación, maximizando la efectividad y costoeficiencia de la cirugía (4)]. Asimismo, es probable la disminución de dolor crónico postoperatorio cuando el dolor agudo después de la cirugía es menos intenso, siendo este un beneficio adicional (므. Usualmente el dolor postoperatorio después de un RTPR es de moderado a intenso (6) y la analgesia multimodal es el estándar de manejo (ㄱ). Su objetivo es reducir el uso de opioides y secundariamente sus efectos adversos, como el estado mental alterado, la somnolencia y la depresión respiratoria [].

Los bloqueos de nervios periféricos guiados por ultrasonografía se han posicionado como medidas seguras y efectivas en el control del dolor postoperatorio, como parte de estrategias de analgesia multimodal (9).
2/10 for both groups. The average pain on movement on the second postoperative day was NRS 5/10 for patients with LIA+ACB and 4/10 for patients with PFC+SNB, $p=0,073$. The percentage of patients who fulfilled $\geq 80 \%$ of the rehabilitation goals was similar in both groups $(p=0,201)$.

Conclusions: In the TKA postoperative analgesic management, both techniques are equivalent during the first 48 hours. Although it is known that the LIA+ACB technique generates less motor impairment of the quadriceps muscle, this was not reflected on better performance during physical rehabilitation, possibly due to insufficient analgesic control at 48 hours.

Key words: Arthroplasty, knee, anesthesia and analgesia, postoperative pain, physical and rehabilitation medicine.
En nuestra institución el uso de técnicas combinadas de anestesia regional con catéter perineural femoral y bloqueo de nervio ciático (CPF + BNC) guiados por ultrasonografía se asoció con menor incidencia de dolor moderado a intenso durante las primeras 48 horas postoperatorias (10), por lo que se consolidó como el manejo analgésico estándar para esta población quirúrgica. De forma adversa, el bloqueo de nervio femoral continuo mediante catéter perineural genera debilidad muscular del cuádriceps (11), por lo que algunos autores lo consideran un factor de riesgo independiente para caídas durante el periodo de recuperación (12). Adicionalmente, el bloqueo del nervio ciático, aunque útil para el control analgésico durante las primeras 24 horas (13), es aún controvertido debido al bloqueo motor concomitante de la pierna y el pie, funcionalidad que es importante para la rehabilitación temprana del paciente (14-16).

Estudios anatómicos recientes han dilucidado los detalles de la inervación sensitiva de la rodilla (17-19), lo que ha llevado a la implementación de procedimientos de anestesia regional más localizada del tipo bloqueo de canal de aductores e infiltración de anestésico local periarticular, que no involucran la inervación motora del músculo cuádriceps y tienen una aparente igual efectividad analgésica (20-2己). Se ha visto con su uso alguna mejoría en el cumplimiento de los objetivos de rehabilitación $(23,24)$, sumado a la disminución del riesgo de caídas (25), por lo que mejora la seguridad en el cuidado del paciente y se logra una reincorporación temprana a su cotidianidad. Asimismo, al comparar esta última combinación con técnicas de bloqueo nervioso femoral y ciático más proximales, se demostró mejor desempeño en la marcha durante el primer 
día postoperatorio, con mejor control analgésico en el contexto de un esquema oral de analgesia multimodal establecido [26].

Por consiguiente, durante el año 2018 se diseñó e implementó en nuestra institución un plan de manejo analgésico multimodal para pacientes llevados a RTPR, el cual incluyó la combinación de infiltración de anestésico local periarticular (IAL) con el bloqueo del canal de aductores guiado por ultrasonografía (BCA), teniendo en cuenta que su combinación presenta mejores resultados que su uso de forma aislada (르). Para evaluar el nuevo protocolo analgésico implementado se compararon retrospectivamente el dolor en reposo y en movimiento durante las primeras 48 horas postoperatorias frente a un grupo similar de pacientes a los que se les realizó RTPR durante el año 2017 y que recibieron dentro de su manejo analgésico postoperatorio infusión continua de anestésico local a través de un catéter perineural femoral (CPF] asociada al bloqueo de nervio ciático en dosis única (BNC), guiados ambos por ultrasonografía. El cumplimiento de las metas de rehabilitación física, evaluado según el esquema institucional, se consideró un objetivo secundario a comparar, pensado como una variable dependiente del adecuado control analgésico.

\section{MATERIALES Y MÉTODOS}

Estudio observacional ambispectivo de cohortes no aleatorizado, realizado en un centro de salud de alta complejidad del suroccidente colombiano. El grupo de pacientes de referencia para realizar la comparación retrospectiva contiene aquellos a los que se les realizó RTPR durante el año 2017 y que recibieron, dentro de su manejo analgésico, catéter femoral perineural con infusión continua de anestésico local durante las primeras 48 horas postoperatorias más bloqueo de nervio ciático dosis única (grupo CPF + BNC). El esquema analgésico intra y postoperatorio fue determinado por el grupo quirúrgico de forma no estandarizada, donde se incluyó el uso de antinflamatorios no esteroides (AINE) y opioides fuertes de rescate en la unidad de cuidado postanestésico (UCPA) y durante la hospitalización subsecuente. El uso de ketamina en el postoperatorio se usó como estrategia de rescate en UCPA en caso de no mejoría después de una titulación adecuada de opioide fuerte según el protocolo de analgesia institucional.

Durante el año 2018 se implementó un protocolo de analgesia multimodal para la evaluación prospectiva de su impacto en el manejo del dolor postoperatorio en pacientes con RTPR que incluyó el uso de bloqueo de canal de aductores dosis única asociado a infiltración de anestésico local periarticular intraoperatoria (grupo IAL + BCA] más un esquema de analgesia multimodal estandarizado durante la fase pre, intra, y postoperatoria, detallado en la Tabla I.

Los datos poblacionales y clínicos de los pacientes se extrajeron de las historias clínicas incluyendo el registro anestésico y las notas de enfermería respectivas para cada sitio de estancia hospitalaria. Los datos poblacionales incluyeron edad, sexo, índice de masa corporal (IMC), diagnóstico, antecedentes personales (que inluyó presencia de comorbilidades], uso regular de analgési- cos orales, otros fármacos neuromoduladores o moduladores de ánimo y escala de Oxford (evalúa severidad de alteración funcional secundario a enfermedad articular] (28). Dentro de los datos clínicos se midió dolor postoperatorio en reposo en UCPA, en hospitalización a las 24 y 48 horas y en movimiento durante la terapia física a las 48 horas como objetivo primario. El uso de opioides intravenosos y número de dosis de rescate, presencia de náuseas y vómitos postoperatorios (NVPO) durante la hospitalización y el cumplimiento de las metas de rehabilitación física a las 48 horas fueron objetivos secundarios.

Para la valoración del dolor se utilizó la escala verbal numérica (EVN), con puntuaciones de 0 a 10 dependiendo de la intensidad del dolor referido. Se consideró dolor leve de 1 a 3 , dolor moderado de 4 a 6 y dolor intenso de 7 a 10. Lo anterior fue evaluado por la clínica de dolor agudo en el seguimiento diario del paciente hospitalizado, con dependencia directa del servicio de anestesiología, que además valoró la realización de actividades básicas de recuperación y la presencia de efectos adversos.

Por otro lado, las metas de rehabilitación temprana para pacientes sometidos a RTPR se encuentran descritas en la guía institucional, realizada por el servicio de fisiatría y fisioterapia. Para la valoración del cumplimiento de metas se definieron ejercicios para el primer y el segundo día postoperatorio. Los ejercicios del primer día se realizaron en cama con la ayuda del acompañante si el paciente así lo requería, e incluyeron movilización de los tobillos, flexionar la rodilla según tolerancia de dolor, elevación del miembro inferior operado, separación de los miembros inferiores y contracción activa de cuádriceps y glúteos. Los ejercicios del segundo día se realizaron de pie con ayuda de un caminador y bajo supervisión, e incluyeron abducción y extensión activa del miembro inferior operado. El objetivo final de la terapia física fue lograr la deambulación asistida con caminador por el pasillo. El grado de cumplimiento se valoró en un porcentaje del 0 \% al $100 \%$ (Tabla II) según las metas alcanzadas.

Para su comparación, se realizó una revisión retrospectiva de los datos mencionados previamente en aquellos pacientes a los que se les realizó RTPR durante el año 2017 y que recibieron dentro de su manejo analgésico el CPF + BNC.

La información fue registrada en una base de datos en formato electrónico con acceso restringido. Se compararon entre los grupos la mediana de valoración de dolor en los diferentes momentos de la medición del objetivo primario. Adicionalmente, se compararon las proporciones de pacientes con dolor leve, moderado o severo, entendiendo como objetivo analgésico ideal presentar dolor leve durante el seguimiento, tanto en reposo como en movimiento. Las proporciones de pacientes que presentaron rescates de opioide parenteral, y la presencia de efectos secundarios durante el total del tiempo de seguimiento, fueron también desenlaces secundarios comparados. Respecto a las metas de rehabilitación física, en conjunto con el grupo de fisiatría y fisioterapia, se consideró como objetivo ideal el cumplimiento de más del $80 \%$ de las metas establecidas, es decir, la deambulación asistida en la habitación de al menos 3 metros. Se compararon entre los grupos las proporciones de pacientes que lograron este objetivo, 
TABLA I

ESQUEMAS ANALGÉSICOS UTILIZADOS EN LA INSTITUCIÓN

\begin{tabular}{|c|c|c|}
\hline Año & 2017 & 2018 \\
\hline Tratamiento & Analgesia estándar & Nuevo protocolo de analgesia multimodal \\
\hline \multirow[t]{2}{*}{$\begin{array}{l}\text { Técnica de anestesia } \\
\text { regional }\end{array}$} & $\begin{array}{l}\text { Catéter perineural femoral guiado } \\
\text { por ultrasonografía: } \\
\text { Bupivacaína } 0,125 \% 6 \mathrm{ml} / \mathrm{h} \\
\text { + PCA } 4 \mathrm{ml} \text { cada } 30 \text { minutos a } \\
\text { necesidad del paciente }\end{array}$ & $\begin{array}{l}\text { Infiltración de anestésico local: en cápsula } \\
\text { posterior, ligamentos colaterales, colgajo } \\
\text { capsular, tejido subcutáneo y bordes de la } \\
\text { herida } \\
\text { Volumen total: } 120 \mathrm{ml} \\
\text { Levobupivacaína } 0,125 \% \text { + adrenalina } 5 \mathrm{mg} / \mathrm{ml} \\
\text { + ketorolaco } 30 \mathrm{mg}\end{array}$ \\
\hline & $\begin{array}{l}\text { Bloqueo del nervio ciático guiado } \\
\text { por ultrasonografía (dosis única) } \\
20 \mathrm{ml} \text { de levobupivacaína } 0,5 \%\end{array}$ & $\begin{array}{l}\text { Bloqueo del canal de aductores guiado por } \\
\text { ultrasonografía (dosis única) } \\
15 \mathrm{ml} \text { de levobupivacaína 0,375 \% }\end{array}$ \\
\hline Preoperatorio & $\begin{array}{l}\text { Técnica anestésica a discreción } \\
\text { del anestesiólogo tratante }\end{array}$ & $\begin{array}{l}30 \text { minutos antes de la cirugía: acetaminofeno } \\
1 \mathrm{~g} \text { v.o. + parecoxib } 40 \text { mg i.v. }\end{array}$ \\
\hline Intraoperatorio & $\begin{array}{l}\text { Antinflamatorios no esteroideos a } \\
\text { discreción del grupo quirúrgico }\end{array}$ & $\begin{array}{l}\text { Técnica de anestesia neuroaxial } \\
\text { (preferiblemente) } \\
\text { Dexametasona } 4 \mathrm{mg} \text { i.v. } \\
\text { Ondansetrón } 4 \mathrm{mg} \text { i.v. } \\
\text { Hidromorfona } 0,01 \mathrm{mg} / \mathrm{kg} \text { i.v. } \\
\text { Ketamina } 0,3 \mathrm{mg} / \mathrm{kg} \text { i.v. (2 dosis) } \\
\text { Dipirona } 1.250 \mathrm{mg} \text { i.v. en caso de } \\
\text { contraindicación al uso de parecoxib }\end{array}$ \\
\hline $\begin{array}{l}\text { Unidad de cuidados } \\
\text { postanestésicos }\end{array}$ & $\begin{array}{l}\text { Opioides fuertes parenterales de } \\
\text { rescate si EVN }>4 \\
\text { Ketamina } 0,3 \mathrm{mg} / \mathrm{kg} \text { (si persiste } \\
\text { dolor después de titulación } \\
\text { adecuada de opioides) }\end{array}$ & $\begin{array}{l}\text { Hidromorfona i.v. titulada si la EVN }>4 \\
\text { Ketamina } 0,3 \mathrm{mg} / \mathrm{kg} \text { (si persiste dolor después } \\
\text { de titulación adecuada de opioides) }\end{array}$ \\
\hline Hospitalización en piso & $\begin{array}{l}\text { Antinflamatorios no esteroideos a } \\
\text { discreción del grupo quirúrgico } \\
\text { Opioide fuerte titulado (rescate } \\
\text { analgésico si EVN > 4) }\end{array}$ & $\begin{array}{l}\text { Acetaminofeno } 1 \mathrm{~g} \text { v.o. cada } 8 \mathrm{~h} \\
\text { Celecoxib } 200 \mathrm{mg} \text { v.o. cada } 12 \mathrm{~h} \\
\text { Hidrocodona } 5 \mathrm{mg} \text { v.o. cada } 8 \mathrm{~h} \\
\text { Hidromorfona i.v. titulada (analgesia de rescate } \\
\text { si EVN > 4) } \\
\text { Dipirona } 1.250 \mathrm{mg} \text { i.v. cada } 6 \text { horas en caso } \\
\text { de contraindicación al uso de celecoxib }\end{array}$ \\
\hline & \multicolumn{2}{|c|}{ El seguimiento diario del dolor postoperatorio lo realiza la clínica de dolor agudo } \\
\hline & \multicolumn{2}{|c|}{ El cumplimiento de las metas de rehabilitación física lo evalúan los fisioterapeutas } \\
\hline
\end{tabular}

EVN: escala verbal numérica; PCA: patient controlled analgesia.

TABLA II

CUMPLIMIENTO DE REHABILITACIÓN FÍSICA TEMPRANA

\begin{tabular}{|l|l|}
\hline $20 \%$ & Realiza ejercicios del primer día y logra posición de sedente largo en la cama \\
\hline $50 \%$ & Realiza ejercicios del primer día y logra transición a sedente en el borde de la cama \\
\hline $60 \%$ & Realiza ejercicios del primer día y logra traslado a silla \\
\hline $80 \%$ & Realiza ejercicios del segundo día y logra marcha en la habitación (prueba de 3 metros) \\
\hline $100 \%$ & Realiza ejercicios del segundo día y marcha en el pasillo (prueba de 6 minutos) \\
\hline
\end{tabular}


y se realizó un análisis diferencial según otras variables clínicas que pudiesen influenciar su desempeño en el proceso de rehabilitación, tales como presencia de dolor moderado a severo, sexo, edad e IMC.

Ambos procesos se realizaron dentro de los principios éticos propuestos en la declaración de Helsinki. Según la Resolución 008430 del Ministerio de Salud Colombiano, este estudio es una investigación sin riesgo, por lo que se solicitó al Comité de Ética Institucional la exención del consentimiento informado. Por medio del Acta número 20 de 4 de octubre de 2017, el Comité de Ética en Investigación Biomédica dio su aprobación al presente estudio (protocolo número 1189).

\section{Análisis estadístico}

Se utilizó estadística descriptiva. La distribución de las variables cuantitativas se calculó con la prueba de Shapiro-Wilk. Los datos con distribución normal se representaron con promedio y desviación estándar, mientras que los datos con distribución que no cumplió criterios de normalidad se describieron con mediana y rango intercuartílico. Por su parte, las variables cualitativas se presentan con frecuencias absolutas y porcentajes.
La comparación de las variables cuantitativas se realizó con la prueba t de Student (variables con distribución normal) o la prueba $U$ de Mann-Whitney (variables con distribución no normal). Para las variables cualitativas se utilizó la prueba $\chi^{2}$ o la prueba exacta de Fisher, según la frecuencia esperada menor a 5 . Se manejó un nivel de significancia del $5 \%$.

\section{RESULTADOS}

Durante el periodo analizado, 120 pacientes fueron llevados a RTPR, sin embargo 8 pacientes fueron excluidos por pérdida de información.

Se analizaron un total de 112 pacientes, de los cuales 67 recibieron analgesia con IAL + BCA y 45 con CPF + BNC. Los grupos fueron comparables en edad, sexo e IMC. En contraste, se identificaron diferencias en antecedentes personales de diabetes mellitus ( $p=0,011)$, uso regular de antiinflamatorios no esteroideos (AINE) $(p<0,01)$ y uso regular de opioides $(p=0,026$ ) (Tabla III).

En la Tabla IV se exponen las condiciones clínicas de los pacientes durante su estancia en la UCPA. Se encontró similitud entre los grupos respecto a intensi-

TABLA III

CARACTERÍSTICAS DEMOGRÁFICAS Y CLÍNICAS DE LOS PACIENTES

\begin{tabular}{|c|c|c|c|}
\hline & Grupo IAL + BCA $(n=67)$ & Grupo CPF + BNC ( $n=45)$ & Valor $\mathrm{p}$ \\
\hline Edad* & 70 (62-76) & 70 (65-75) & 0,842 \\
\hline Sexo femenino, n [\%] & $47(70,2)$ & $30(66,7)$ & 0,697 \\
\hline Índice de masa corporal & $29,3(26,3-32,8)$ & $28,9(26,6-32,4)$ & 0,856 \\
\hline \multicolumn{4}{|l|}{ Diagnóstico principal, n [\%] } \\
\hline Artrosis & $60(89,6)$ & $42(93,3)$ & \multirow{2}{*}{0,741} \\
\hline Artritis & $7(10,4)$ & $3(6,7)$ & \\
\hline Escala de Oxford * & $14(10-18)$ & $14(12-20)$ & 0,183 \\
\hline \multicolumn{4}{|l|}{ Antecedentes patológicos, $\mathrm{n}$ [\%] } \\
\hline Diabetes mellitus & $17(25,4)$ & $3(6,7)$ & 0,011 \\
\hline Enfermedad cardiovascular & $51(76,1)$ & $28(62,2)$ & 0,114 \\
\hline Enfermedad renal crónica & 6 (9) & $2(4,4)$ & 0,472 \\
\hline Enfermedad cerebrovascular & $4[6]$ & $1(2,2)$ & 0,646 \\
\hline Ansiedad-depresión & $5(7,5)$ & $0(0)$ & - \\
\hline \multicolumn{4}{|l|}{ Antecedentes farmacológicos, n [\%] } \\
\hline Uso regular de AINE & $36(53,7)$ & $7(15,6)$ & $<0,01$ \\
\hline Uso regular de opioides & $15(22,4)$ & $3(6,7)$ & 0,026 \\
\hline Uso regular de gabapentinoides & $5(7,5)$ & $0(0)$ & - \\
\hline Uso regular de antidepresivos duales & 2 (3) & $0(0)$ & - \\
\hline Uso regular de ISRS & $4(6)$ & $1(2,2)$ & 0,646 \\
\hline Uso regular de benzodiacepinas & $1(1,5)$ & $1(2,2)$ & 1 \\
\hline
\end{tabular}

*Mediana (rango intercuartílico). AINE: antinflamatorios no esteroideos. CPF + BNC: catéter perineural femoral más bloqueo del nervio ciático. IAL + BCA: infiltración de anestésico local periarticular más bloqueo del canal de aductores. ISRS: inhibidores selectivos de la recaptación de serotonina. NA: no aplica. 
dad del dolor, requerimiento de ketamina y presencia de NVPO. La proporción de pacientes que usaron opioides fue mayor en el grupo con IAL + BCA [25 [37,3\%] vs. CPF + BNC 6 [13,3\%]; $p<0,01]$; sin embargo, la mediana del número de dosis requeridas de opioides por paciente fue mayor en el grupo con CPF + BNC [3 [2-4] vs. IAL + BCA: 1 [1-1]; $p<0,01]$.

Durante la hospitalización en planta, la mediana del dolor en reposo en el primer y segundo día postoperatorio fue $2 / 10$ para ambos grupos, con valor $p=0,25$ y $p=0,463$ respectivamente. El promedio del dolor en movimiento en el segundo día postoperatorio fue 5/10 para los pacientes con IAL + BCA y 4/10 para los pacientes con CPF + BNC, $p=0,073$. Respecto al uso de opioides, la proporción fue mayor en el grupo con $\mathrm{IAL}+\mathrm{BCA}[53[79,1 \%]$ vs. CPF + BNC $23[51,1 \%]$ $p<0,01$ ), al igual que el número de dosis de rescate con opiodes con IAL + BCA [2 [ \pm 1$]$ vs. CPF + BNC $1[ \pm 1]$; $p<0,01$ ]. No hubo diferencia estadísticamente significativa en la proporción de pacientes con NVPO (Tabla V).

En lo referente al cumplimiento de las metas de rehabilitación física, en ambos grupos se tiene una proporción similar de pacientes con cumplimiento del $80 \%$ o más de la terapia $(p=0,201)$. Este mismo comportamiento se observó al estratificar por sexo, edad, IMC y dolor en reposo a las 48 horas (Tabla VI).

TABLA IV

VARIABLES CLIINICAS DE LOS PACIENTES EN LA UNIDAD DE CUIDADOS POSTANESTÉSICOS

\begin{tabular}{|l|c|c|c|}
\hline & $I A L+B C A(n=67)$ & $C P F+B N C(n=45)$ & Valor $p$ \\
\hline Dolor en reposo & $57(85,1)$ & $40(88,9)$ & 0,561 \\
\hline Leve (EVN 0-3) & $10(14,9)$ & $5(11,1)$ & $<0,01$ \\
\hline Intenso (EVN 7-10) & $25(37,3)$ & $6(13,3)$ & $<0,01$ \\
\hline Uso de opioides & $1(1-1)$ & $3(2-4)$ & - \\
\hline Número de dosis de opioides* & $0(0)$ & $1(2,2)$ & 0,648 \\
\hline Uso de ketamina & $3(4,5)$ & $1(2,2)$ & \\
\hline NVPO &
\end{tabular}

*Mediana (rango intercuartílico). CPF + BNC: catéter perineural femoral más bloqueo del nervio ciático. EVN: escala visual numérica. IAL + BCA: infiltración de anestésico local periarticular más bloqueo del canal de aductores. NVPO: náuseas y vómitos postoperatorios.

TABLA V

VARIABLES CLÍNICAS DE LOS PACIENTES DURANTE LA HOSPITALIZACIÓN EN PLANTA

\begin{tabular}{|c|c|c|c|}
\hline & Grupo IAL + BCA $(n=67)$ & Grupo $C P F+B N C(n=45)$ & Valor $\mathrm{p}$ \\
\hline \multicolumn{4}{|c|}{ Dolor en reposo día 1 postoperatoio, $n$ [\%] } \\
\hline Leve [EVN 0-3] & $56(83,6]$ & $35(77,8)$ & \multirow{3}{*}{0,344} \\
\hline Moderado (EVN 4-6) & $10(14,9)$ & $7(15,5)$ & \\
\hline Intenso (EVN 7-10) & $1(1,5)$ & $3(6,7)$ & \\
\hline \multicolumn{4}{|c|}{ Dolor en reposo día 2 postoperatorio, n [\%] } \\
\hline Leve (EVN 0-3) & $54(80,6)$ & $40(88,9)$ & \multirow{3}{*}{0,4} \\
\hline Moderado (EVN 4-6) & $12(17,9)$ & $4(8,9)$ & \\
\hline Intenso (EVN 7-10) & $1(1,5)$ & $1(2,2)$ & \\
\hline \multicolumn{4}{|c|}{ Dolor en movimiento día 2 postoperatorio, $\mathrm{n}$ [\%] } \\
\hline Leve (EVN 0-3] & $20(29,9)$ & $19(42,2)$ & \multirow{3}{*}{0,372} \\
\hline Moderado (EVN 4-6) & $36(53,7)$ & $21(46,7)$ & \\
\hline Intenso (EVN 7-10) & $11(16,4)$ & $5(11,1)$ & \\
\hline Uso de opioides & $53(79,1)$ & $23(51,1)$ & $<0,01$ \\
\hline $\begin{array}{l}\text { Número de dosis de opioides día } 1 \\
\text { postoperatorio* }\end{array}$ & $2[ \pm 1]$ & $1( \pm 1)$ & $<0,01$ \\
\hline NVPO & $7(10,5)$ & $1(2,2)$ & 0,141 \\
\hline
\end{tabular}

*Promedio (desviación estándar). CPF + BNC: catéter perineural femoral más bloqueo del nervio ciático. EVN: escala visual numérica. IAL + BCA: infiltración de anestésico local periarticular más bloqueo del canal de aductores. NVPO: náuseas y vómitos postoperatorios. 
TABLA VI

CUMPLIMIENTO DE METAS DE REHABILITACIÓN FÍSICA

\begin{tabular}{|c|c|c|c|}
\hline & Grupo IAL + BCA $(n=67)$ & Grupo CPF +BNC ( $n=45)$ & Valor $\mathrm{p}$ \\
\hline \multicolumn{4}{|c|}{ Cumplimiento general } \\
\hline$<80 \%$ & $35(52,2)$ & $29(64,4)$ & \multirow{2}{*}{0,201} \\
\hline$\geq 80 \%$ & $32[47,8]$ & $16(35,6)$ & \\
\hline \multicolumn{4}{|c|}{ Clasificación del dolor en reposo día 2 postopertorio } \\
\hline \multicolumn{4}{|l|}{ Leve } \\
\hline$<80 \%$ & 27 (50) & $25(62,5)$ & \multirow{2}{*}{ 0,228 } \\
\hline$\geq 80 \%$ & $27(50)$ & $15(37,5)$ & \\
\hline \multicolumn{4}{|c|}{ Moderado a intenso } \\
\hline$<80 \%$ & $8(61,5)$ & $4(80)$ & \multirow{2}{*}{0,615} \\
\hline$\geq 80 \%$ & $5(38,5)$ & $1(20)$ & \\
\hline \multicolumn{4}{|l|}{ Sexo } \\
\hline \multicolumn{4}{|l|}{ Femenino } \\
\hline$<80 \%$ & $26(55,3)$ & $18(60)$ & \multirow{2}{*}{0,686} \\
\hline$\geq 80 \%$ & $21(44,7)$ & $12(40)$ & \\
\hline \multicolumn{4}{|l|}{ Masculino } \\
\hline$<80 \%$ & $9(45)$ & $11(73,3)$ & \multirow{2}{*}{0,094} \\
\hline$\geq 80 \%$ & $11(55)$ & $4(26,7)$ & \\
\hline \multicolumn{4}{|c|}{\begin{tabular}{|l|} 
Grupo etario \\
\end{tabular}} \\
\hline \multicolumn{4}{|c|}{$\leq 59$ años } \\
\hline$<80 \%$ & $9(75)$ & $3(60)$ & \multirow{2}{*}{0,6} \\
\hline$\geq 80 \%$ & $3(25)$ & $2(40)$ & \\
\hline \multicolumn{4}{|c|}{$\geq 60$ años } \\
\hline$<80 \%$ & $26(47,3)$ & 26 (65) & \multirow{2}{*}{0,087} \\
\hline$\geq 80 \%$ & $29(52,7)$ & 14 (35) & \\
\hline \multicolumn{4}{|c|}{ Índice de masa corporal } \\
\hline \multicolumn{4}{|l|}{ Normal } \\
\hline$<80 \%$ & $5(62,5)$ & $3(60)$ & \multirow{2}{*}{1} \\
\hline$\geq 80 \%$ & $3(37,5)$ & $2(40)$ & \\
\hline \multicolumn{4}{|c|}{\begin{tabular}{|l|} 
Sobrepeso \\
\end{tabular}} \\
\hline$<80 \%$ & $17(54,8)$ & $14(60,9)$ & \multirow{2}{*}{0,658} \\
\hline$\geq 80 \%$ & $14(45,2)$ & $9(39,1)$ & \\
\hline \multicolumn{4}{|l|}{ Obesidad } \\
\hline$<80 \%$ & $13(46,4)$ & $12(70,6)$ & \multirow{2}{*}{0,114} \\
\hline$\geq 80 \%$ & $15(53,6)$ & $5(29,4)$ & \\
\hline
\end{tabular}

CPF + BNC: catéter perineural femoral más bloqueo del nervio ciático. IAL + BCA: infiltración de anestésico local periarticular más bloqueo del canal de aductores.

\section{DISCUSIÓN}

En este trabajo se hace una comparación de dos estrategias analgésicas para el manejo del dolor postoperatorio en pacientes a quienes se les realizó RTPR. Se evidenció que la respuesta analgésica al esquema multimodal establecido en el grupo $\mathrm{IAL}+\mathrm{BCA}$ es similar al grupo CPF + BNC al no encontrarse diferencias significativas en la evaluación del dolor en reposo ni en movimiento durante su estancia en UCPA, y durante las primeras 48 horas de hospitalización postquirúrgica. Estos hallazgos son acordes a estudios previos similares $(25,29)$. Sin embargo, los pacientes del grupo de IAL + BCA requirieron mayor uso de opioide intravenoso en hospitalización, aunque no es un factor comparable al no estar incluido en el plan de manejo de forma predefinida en el grupo CPF + BNC. De forma similar, en UCPA fueron más pacientes los que requirieron opioides de 
rescate en el grupo de IAL+BCA, hallazgo similar a los encontrados en otros estudios [21]).

En términos generales los grupos estudiados fueron equivalentes y comparables respecto a las características demográficas y clínicas. Se encontró diferencia estadísticamente significativa en el antecedente de diabetes mellitus en el grupo de IAL + BCA; sin embargo, la relación causal con el desenlace de interés no se considera significativa (30). Asimismo, se encontró mayor uso regular preoperatorio de AINE y opioides en este grupo, aunque podría resultar secundario a un sesgo de información ante la diferencia en la obtención de la misma entre los grupos, la cual fue prospectiva en el grupo de los pacientes sometidos a IAL + BCA y retrospectiva de las historias clínicas en el grupo de control.

Se confirma, acorde con estudios previos $(25,29)$, que ambas estrategias analgésicas son equivalentes, pues no hay diferencias significativas entre los grupos en la evaluación del dolor en reposo ni en movimiento, con un perfil similar de efectos adversos. Los pacientes del grupo de IAL + BCA requirieron mayor uso de opioides intravenosos en hospitalización, aunque no es un factor comparable al no estar incluido en el plan de manejo de forma predefinida en el grupo control, como sí lo estuvo en el protocolo aplicado en el grupo de intervención. De forma similar, en la UCPA fueron más pacientes los que requirieron opioides de rescate en el grupo de IAL + BCA, hallazgo similar a los encontrados en otros estudios [21].

Al evaluar el cumplimiento de los objetivos en las metas de rehabilitación a las 48 horas, se encontró que no hubo diferencia a favor de ningún grupo, contradictoriamente a la naturaleza de la intervención, que promueve el mantenimiento de la fuerza del músculo cuádriceps (31), lo que pudiera reflejarse en un mejor desempeño durante la deambulación como objetivo final de la rehabilitación. En diversos estudios el rango de movimiento, la fuerza del músculo cuádriceps y la habilidad en la movilización y deambulación son mayores en las primeras 24 horas a favor del BCA frente al bloqueo del nervio femoral (BNF) $(25,32,33)$. Debido a que nuestras metas de rehabilitación fueron medidas a las 48 horas, los hallazgos en el presente estudio no son comparables con la información previa. Es posible que con el paso del tiempo, una vez finalizado el efecto del anestésico local del BCA posterior a las primeras 24 horas, el dolor reincidente de intensidad moderada durante la movilización limite el cumplimiento de los objetivos de rehabilitación. Esta teoría se soporta en el estudio realizado por Macrinici y cols. (34), en el cual se evaluaron dos grupos de pacientes que recibieron uno BNF y el otro BCA, para el manejo de dolor posterior a la realización de RTPR; en este segundo grupo se identificó una disminución progresiva del porcentaje de fuerza preservada en el cuádriceps tras el BCA a medida que la intensidad de dolor iba aumentando, lo que posiblemente explica por qué a las 48 horas no hubo diferencia en la prueba Timed Up and Go como medida objetiva de la capacidad para la deambulación.

Aunque hay datos recientes que sugieren que el postergar la infusión continua de anestésico local a través de un catéter en el canal de aductores por 48 horas no representa ventajas en la prevención del dolor postoperatorio intenso comparado con el BCA en dosis única preoperatoria, sí hay una mayor proporción de pacientes con dolor leve y moderado (35). Otros datos muestran que aunque el BNF y el BCA continuos son equivalentes en cuanto a control analgésico y consumo de opioides durante las primeras 48 horas, los pacientes con BCA continuo lograron realizar la prueba Timed Up and Go y mínimo 30 metros de marcha más tempranamente de forma significativa (25 vs. 42 horas] (36), sin que eso signifique un alta más temprana al tener los mismos requerimientos de opioides fuertes intravenosos.

Confirmamos con este estudio que en nuestra práctica clínica el uso de protocolos de analgesia multimodal con técnicas sencillas de anestesia regional, como lo es el BCA dosis única más IAL periarticular, permite un control adecuado del dolor en reposo durante las primeras 48 horas, equivalente al uso de técnicas avanzadas como lo son el CPF y el BNC proximal que requieren para su realización anestesiólogos con mayor experticia y el uso de instrumentos más especializados (37). Aunque los datos no son concluyentes, hay una tendencia a un mayor consumo y requerimiento de opioides adicionales durante el periodo postoperatorio mediato en el grupo BCA + IAL, sin que esto signifique mayor incidencia de efectos secundarios. Dada la simplicidad y equivalencia analgésica puede facilitarse su aplicación de forma universal como parte de guías clínicas estandarizadas.

Es importante resaltar que el dolor postoperatorio en movimiento a las 48 horas fue moderado (EVN 4-6) en aproximadamente la mitad de la población en ambos grupos, el cual puede ser un factor determinante en el incumplimiento de las metas de rehabilitación postoperatoria propuestas, lo que pudiese orientar hacia la optimización de los esquemas de analgesia multimodal con opciones tales como la inclusión de opioides de liberación prolongada (38) o neuromoduladores tipo duloxetina o pregabalina que, aunque controvertidos, han mostrado ser útiles en algunos esquemas evaluados $(39,40)$, en particular al considerar algunos hallazgos experimentales que evidencian sensibilización central en pacientes con dolor severo por osteoartrosis por una menor tolerancia a la presión y temperatura extremos, al compararse con controles sanos y pacientes con osteoartrosis con dolor no severo. La sensibilización central, en parte causada por la alteración de los sistemas de control descendente generados por la presencia de dolor crónico, se ha identificado como causa de la hiperalgesia presente en los tejidos circundantes a la articulación de la rodilla en pacientes con osteoartritis (41). Adicionalmente, el consumo previo de opioides, aun a bajas dosis, en pacientes con patologías osteoarticulares crónicas, se ha relacionado con la presencia de hiperalgesia y un mayor consumo de opioides en el periodo postoperatorio (42). Es posible que la identificación de pacientes con sensibilización central antes del procedimiento pueda orientar el uso de neuromoduladores y disminuir la hiperalgesia postoperatoria, siendo este un posible campo de investigación futura.

A pesar de ser un estudio observacional, no aleatorizado, con posibilidad de sesgos en su desarrollo que pueden limitar la validez interna de los resultados, este estudio se desarrolla en una población representativa y cada vez más frecuente de pacientes susceptibles de 
RTPR. A partir de este estudio se establece la necesidad de identificar las posibles estrategias que fomenten la prolongación de la analgesia después de 24 horas postoperatorias, con menor compromiso motor y mejor desempeño en las actividades de rehabilitación.

\section{CONFLICTO DE INTERESES}

Los autores declaran no tener conflicto de intereses.

\section{AGRADECIMIENTOS}

Agradecemos al Centro de Investigaciones Clínicas de la Fundación Valle del Lili, especialmente a Diana Marcela Martínez Ruiz, por su colaboración con la sección de metodología. También a Diana Marcela Loaiza Ruiz, MD, por su contribución en la redacción de la discusión. Finalmente, agradecemos al grupo de ortopedistas y anestesiólogos de la Fundación Valle del Lili por su cooperación con la implementación del protocolo.

\section{FINANCIACIÓN}

Este estudio fue financiado con recursos de la Fundación Valle del Lili.

\section{BIBLIOGRAFÍA}

1. Thomsen MG, Husted H, Otte KS, Orsnes T, Troelsen A. Indications for knee arthroplasty have remained consistent over time. Dan Med J. 2012;59(8):A4492.

2. Carr AJ, Robertsson O, Graves S, Price AJ, Arden NK, Judge A, et al. Knee replacement. Lancet. 2012; 379(9823):133140. DOI: 10.1016/S0140-6736(11)60752-6.

3. Inacio MCS, Paxton EW, Graves SE, Namba RS, Nemes S. Projected increase in total knee arthroplasty in the United States-an alternative projection model. Osteoarthritis Cartilage. 2017;25(11):1797-803. DOI: 10.1016/j. joca.2017.07.022.

4. Kumar L, Kumar AH, Grant SA, Gadsden J. Updates in enhanced recovery pathways for total knee arthroplasty. Anesthesiol Clin. 2018;36:375-86. DOI: 10.1016/j. anclin.2018.04.007.

5. Buvanendran A, Della Valle CJ, Kroin JS, Shah M, Moric M, Tuman KJ, et al. Acute postoperative pain is an independent predictor of chronic postsurgical pain following total knee arthroplasty at 6 months: a prospective cohort study. Reg Anesth Pain Med. 2019;44:e100036. DOI: 10.1136/ rapm-2018-100036.

6. Gerbershagen HJ, Aduckathil S, van Wijck AJ, Peelen LM, Kalkman CJ, Meissner W. Pain intensity on the first day after surgery: a prospective cohort study comparing 179 surgical procedures. Anesthesiology. 2013;118(4):934-44. DOI: 10.1097/ALN.Ob013e31828866b3.

7. Elmallah RK, Chughtai M, Khlopas A, Newman JM, Stearns KL, Roche M, et al. Pain control in total knee arthroplasty. J Knee Surg. 2018;31(6):504-13. DOl: 10.1055/s-0037-1604152.

8. Wheeler M, Oderda GM, Ashburn MA, Lipman AG. Adverse events associated with postoperative opioid analgesia: a systematic review. J Pain. 2002;3(3):159-80. DOI: 10.1054/ jpai.2002.123652.
9. Terkawi AS, Mavridis D, Sessler DI, Nunemaker MS, Doais KS, Terkawi RS, et al. Pain management modalities after total knee arthroplasty: a network meta-analysis of 170 randomized controlled trials. Anesthesiology. 2017;126(5):92337. DOI: 10.1097/ALN.0000000000001607.

10. Dorado-Velasco FC, Satizábal-Padridín N. Efficacy of different multimodal analgesia techniques to prevent moderate to severe pain in primary total knee arthroplasty. Rev Colomb Anestesiol. 2018;46:8-16. DOI: 10.1097/ CJ9.0000000000000039.

11. Sharma S, lorio R, Specht LM, Davies-Lepie S, Healy WL. Complications of femoral nerve block for total knee arthroplasty. Clin Orthop Relat Res. 2010;468(1):135-40. DOI: 10.1007/s11999-009-1025-1.

12. Wasserstein D, Farlinger C, Brull R, Mahomed N, Gandhi R. Advanced age, obesity and continuous femoral nerve blockade are independent risk factors for inpatient falls after primary total knee arthroplasty. J Arthroplasty. 2013;28(7):1121-4. DOI: 10.1016/j.arth.2012.08.018.

13. Rojas NE, Gómez RJ, Hamaji A, Hamaji MW, Vieira JE. Continuous femoral nerve blockade and single-shot sciatic nerve block promotes better analgesia and lower bleeding for total knee arthroplasty compared to intrathecal morphine: a randomized trial. BMC Anesthesiol. 2017;17:64. DOI: 10.1186/s12871-017-0355-x.

14. Abdallah FW, Brull R. Is sciatic nerve block advantageous when combined with femoral nerve block for postoperative analgesia following total knee arthroplasty? A systematic review. Reg Anesth Pain Med. 2011;36(5):493-8. DOI: 10.1097/AAP.Ob013e318228d5d4.

15. Grape S, Kirkham KR, Baeriswyl M, Albrecht E. The analgesic efficacy of sciatic nerve block in addition to femoral nerve block in patients undergoing total knee arthroplasty: a systematic review and meta-analysis. Anaesthesia 2016;71(10):1198-209. DOI: 10.1111/anae.13568.

16. Ilfeld BM, Madison SJ. The sciatic nerve and knee arthroplasty: to block, or not to block-that is the question. Reg Anesth Pain Med. 2011;36[5]:421-3. DOI: 10.1097/ AAP.Ob013e31822940d2.

17. Burckett-St Laurant D, Peng P, Girón Arango L, Niazi AU, Chan VW, Agur A, et al. The Nerves of the Adductor Canal and the Innervation of the Knee: An Anatomic Study. Reg Anesth Pain Med. 2016;41(3):321-7. DOI: 10.1097/ AAP.0000000000000389.

18. Anagnostopoulou S, Anagnostis G, Saranteas T, Mavrogenis AF, Paraskeuopoulos T. Saphenous and Infrapatellar Nerves at the Adductor Canal: Anatomy and Implications in Regional Anesthesia. Orthopedics. 2016;39(2):e259-62. DOI: 10.3928/01477447-20160129-03.

19. Tran J, Peng PWH, Lam K, Baig E, Agur AM, Gofeld M. Anatomical Study of the Innervation of Anterior Knee Joint Capsule: Implication for Image-Guided Intervention. Reg Anesth Pain Med. 2018;43(4):407-14. DOI: 10.1097/ AAP.0000000000000778.

20. Macrinici GI, Murphy C, Christman L, Drescher M, Hughes $B$, Macrinici V, et al. Prospective, double-blind, randomized study to evaluate single-injection adductor canal nerve block versus femoral nerve block: postoperative functional outcomes after total knee arthroplasty. Reg Anesth Pain Med. 2017;42(1):10-6. DOI: 10.1097/ AAP.0000000000000507.

21. Amundson AW, Johnson RL, Abdel MP, Mantilla CB, Panchamia JK, Taunton MJ, et al. A three-arm randomized clinical trial comparing continuous femoral plus single-injection sciatic peripheral nerve blocks versus periarticular injection with 
ropivacaine or liposomal bupivacaine for patients undergoing total knee arthroplasty. Anesthesiology. 2017;126(6):113950. DOl: 10.1097/ALN.0000000000001586.

22. Guild GN 3rd, Galindo RP, Marino J, Cushner FD, Scuderi GR. Periarticular regional analgesia in total knee arthroplasty: a review of the neuroanatomy and injection technique. Orthop Clin North Am. 2015;46(1):1-8. DOl: 10.1016/j. ocl.2014.09.016.

23. Ellis TA 2nd, Hammoud H, Dela Merced P, Nooli NP, Ghoddoussi F, Kong J, et al. Multimodal clinical pathway with adductor canal block decreases hospital length of stay, improves pain control, and reduces opioid consumption in total knee arthroplasty patients: a retrospective review. J Arthroplasty. 2018;33(8):2440-8. DOI: 10.1016/j. arth.2018.03.053.

24. Drew JM, Neilio J, Kunze L. Contemporary perioperative analgesia in total knee arthroplasty: multimodal protocols, regional anesthesia, and peripheral nerve blockade. J Knee Surg. 2018;31(7):600-4. DOI: 10.1055/s-00381636835.

25. Wang D, Yang Y, Li Q, Tang SL, Zeng WN, Xu J, et al. Adductor canal block versus femoral nerve block for total knee arthroplasty: a meta-analysis of randomized controlled trials. Sci Rep. 2017;7:40721. DOI: 10.1038/ srep40721.

26. Perlas A, Kirkham KR, Billing R, Tse C, Brull R, Gandhi $R$, et al. The impact of analgesic modality on early ambulation following total knee arthroplasty. Reg Anesth Pain Med. 2013;38(4):334-9. DOI: 10.1097/ AAP.Ob013e318296b6aO.

27. Ma J, Gao F, Sun W, Guo W, Li Z, Wang W. Combined adductor canal block with periarticular infiltration versus periarticular infiltration for analgesia after total knee arthroplasty. Medicine (Baltimore). 2016;95(52):e5701.

28. Dawson J, Fitzpatrick R, Murray D, Carr A. Questionnaire on the perceptions of patients about total knee replacement. J Bone Joint Surg Br. 1998;80(1):63-9. DOI: 10.1302/0301-620X.80B1.0800063.

29. Ludwigson JL, Tillmans SD, Galgon RE, Chambers TA, Heiner JP, Schroeder KM. A Comparison of single shot adductor canal block versus femoral nerve catheter for total knee arthroplasty. J Arthroplasty. 2015; 30(9):68-71. DOI: 10.1016/j.arth.2015.03.044.

30. Wu CL, Raja SN. Treatment of acute postoperative pain. Lancet. 2011;377(9784):2215-2225. DOI: 10.1016/ S0140-6736(11)60245-6.

31. Sørensen JK, Jæger P, Dahl JB, Gottschau B, Stephensen SL, Grevstad $U$. The isolated effect of adductor canal block on quadriceps femoris muscle strength after total knee arthroplasty: a triple-blinded, randomized, placebo-controlled trial with individual patient analysis. Anesth Analg. 2016;122(2):5538. DOI: 10.1213/ANE.0000000000001073.

32. Gao F, Ma J, Sun W, Guo W, Li Z, Wang W. Adductor canal block versus femoral nerve block for analgesia after total knee arthroplasty: a systematic review and meta- analysis. Clin J Pain. 2017;33(4):356-68. DOI: 10.1097/ AJP.0000000000000402.

33. Tan Z, Kang P, Pei F, Shen B, Zhou Z, Yang J. A comparison of adductor canal block and femoral nerve block after totalknee arthroplasty regarding analgesic effect, effectiveness of early rehabilitation, and lateral knee pain relief in the early stage. Medicine (Baltimore). 2018;97(48):e13391. DOI: 10.1097/MD.0000000000013391.

34. Macrinici GI, Murphy C, Christman L, Drescher M, Hughes $B$, Macrinici V, et al. Prospective, double-blind, randomized study to evaluate single-injection adductor canal nerve block versus femoral nerve block: postoperative functional outcomes after total knee arthroplasty. Reg Anesth Pain Med. 2017;42(1):10-6. DOI: 10.1097/ AAP.0000000000000507.

35. Elkassabany NM, Cai LF, Badiola I, Kase B, Liu J, Hughes $\mathrm{C}$, et al. A prospective randomized open-label study of single injection versus continuous adductor canal block for postoperative analgesia after total knee arthroplasty. Bone Joint J. 2019;101-B(3):340-7. DOI: 10.1302/0301-620X.101B3. BJJ-2018-0852.R2.

36. Machi AT, Sztain JF, Kormylo NJ, Madison SJ, Abramson WB, Monahan AM, et al. Discharge readiness after tricompartment knee arthroplasty, adductor canal versus femoral continuous nerve blocks-a dual-center, randomized trial. Anesthesiology. 2015;123(2):444-56. DOI: 10.1097/ ALN.0000000000000741.

37. Sites BD, Chan VW, Neal JM, Weller R, Grau T, Koscielniak-Nielsen ZJ, et al. The American Society of Regional Anesthesia and Pain Medicine and the European Society Of Regional Anaesthesia and Pain Therapy Joint Committee recommendations for education and training in ultrasound-guided regional anesthesia. Reg Anesth Pain Med. 2009;34(1):40-6. DOI: 10.1097/ AAP.Ob013e3181926779.

38. Cheung CW, Ching Wong SS, Qiu Q, Wang X. Oral Oxycodone for Acute Postoperative Pain: A Review of Clinical Trials. Pain Physician. 2017;20(2S):SE33-SE52.

39. Buvanendran A, Kroin J, Della Valle CJ, Kari M, Moric M, Tuman KJ. Perioperative Oral Pregabalin Reduces Chronic. Pain After Total Knee Arthroplasty: A Prospective, Randomized, Controlled Trial. Anesth Analg. 2010;110(1):199207. DOI: 10.1213/ANE.Ob013e3181c4273a.

40. Wang ZY, Shi SY, Li SJ, Chen F, Chen H, Lin HZ, et al. Efficacy and Safety of Duloxetine on Osteoarthritis Knee Pain: A Meta-Analysis of Randomized Controlled Trials. Pain Med. 2015;16(7):1373-85. DOI: 10.1111/pme.12800.

41. Arendt-Nielsen L, Nie H, Laursen MB, Laursen BS, Madeleine $\mathrm{P}$, Simonsen $\mathrm{OH}$, et al. Sensitization in patients with painful knee osteoarthritis. Pain. 2010;149(3):573-81. DOI: 10.1016/j.pain.2010.04.003.

42. Hina N, Fletcher D, Poindessous-Jazat F, Martinez V. Hyperalgesia induced by low-dose opioid treatment before orthopaedic surgery: An observational case-control study. Eur J Anaesthesiol. 2015;32(4):255-61. 